Article

\title{
Energy-Efficient Path Selection Using SNR Correlation for Wireless Multi-Hop Cooperative Communications
}

\author{
In-Ho Lee ${ }^{1}\left(\mathbb{D}\right.$ and Haejoon Jung ${ }^{2, *} \mathbb{D}$ \\ 1 Department of Electrical, Electronic and Control Engineering, Hankyong National University, \\ Anseong 17579, Korea; ihlee@hknu.ac.kr \\ 2 Department of Information and Telecommunication Engineering, Incheon National University, \\ Incheon 22012, Korea \\ * Correspondence: haejoonjung@inu.ac.kr; Tel.: +82-32-835-8631
}

Received: 21 September 2018; Accepted: 31 October 2018; Published: 1 November 2018 updates

\begin{abstract}
In this paper, we consider partial path selection (PPS) for a multi-hop decode-and-forward cooperative system with limited channel state information (CSI) feedback, where the PPS utilizes local CSI only for a subset of hops on each of all independent paths between a source and a destination to reduce the energy consumption for CSI feedback. To enhance the end-to-end performance of the PPS, we propose a novel PPS method with local CSI chosen by the correlation between the end-to-end signal-to-noise ratios (SNRs) based on global and local CSI under Nakagami- $m$ fading channels. For each path, we pick a subset of hops to report CSI with the highest correlation for a given CSI feedback overhead requirement, which can achieve the similar end-to-end outage probability to the best path selection with global CSI. We provide an exact and closed-form expression for the SNR correlation coefficient and present an impact of the SNR correlation on the end-to-end outage probability.
\end{abstract}

Keywords: multi-hop decode-and-forward relaying; limited channel feedback; SNR correlation; relay selection; Nakagami fading channels

\section{Introduction}

Wireless sensor networks (WSNs) have the advantages of high flexibility in deployment and high usability in dynamic environments [1,2]. Because of these advantages, WSNs enable developing diverse applications such as security surveillance, environmental monitoring, disaster monitoring, healthcare, target tracking, and internet of things (IoT) applications [1-4]. However, to realize the long-range communication applications, a limited communication range of WSNs with low-power transmitters should be improved. Thus, multi-hop transmission techniques have been introduced into WSNs for coverage extension. The multi-hop communication allows intermediate nodes between a source node and a destination node to relay a signal of the source node, which can enable long-range communications [5-7].

In WSNs, the nodes may have insufficient space to be equipped with multiple antennas, and thus they may exploit very limited spatial diversity to enhance the signal reliability and data rate. In many studies, hence the concept of virtual antennas has been employed to generate and exploit more spatial diversity, where multiple cooperative nodes are regarded as virtual antennas of a transmitter or a receiver. Such virtual antennas have been also adopted in multi-hop WSNs so as to improve their signal reliability and data rate [8-10]. One of well-known multi-hop cooperation techniques using virtual antennas is the best path selection (BPS) in which a single path providing the best end-to-end channel quality is selected among multiple paths between a source node and a destination node for data 
transmission [11]. The BPS requires global channel state information (CSI) (i.e., full CSI for all hops of every path) for the best path selection. Thus, the BPS may not be employed in wireless communication networks with the limited CSI feedback which can be adopted for the restricted resources such as bandwidth and node energy.

In wireless communication, one of limited resources is energy which can be mainly consumed by signal (e.g., data and control information) transmission and signal processing. The energy resource is significantly treated in a sensor node because of its small energy capability. Such an important energy issue has been also handled in wireless cellular networks to realize the green communication network, and limited CSI feedback has been considered as one of energy-efficient radio technologies [12-15]. Hence, the partial path selection (PPS) utilizing local CSI, i.e., CSI for certain hops of each path, has been proposed in [16]. To enhance the limited end-to-end performance of the PPS, an efficient PPS scheme has been presented in [17], where only CSI for the hop with the smallest average channel power in each dual-hop path is used for the PPS. In addition, the end-to-end performances of the PPS have been widely studied for a decode-and-forward (DF) relaying system with an eavesdropper [18], a cognitive amplify-and-forward (AF) relaying system [19], and a non-orthogonal multiple access (NOMA)-based AF relaying system [20] in terms of the outage probability and ergodic capacity. Moreover, the PPS can be applicable to a cooperative NOMA system [21] and a relay-assisted multicast system [22] when those systems require limited CSI feedback.

However, the PPS techniques in the previous works assuming the dual-hop scenario cannot be directly applied to the multi-hop scenario since many different combinations of hops for local CSI exist in the multi-hop case. Therefore, in this paper, we propose a novel PPS algorithm for a multi-hop relaying system with the limited CSI feedback, by which we can choose a pertinent subset of hops in each path to report local CSI according to the system requirement for CSI feedback overhead. To minimize the performance degradation relative to the BPS based on the global CSI, we introduce the correlation coefficient between the end-to-end signal-to-noise ratios (SNRs) calculated by global and local CSI for each path, and find the subset of hops for local CSI in each path that maximizes the correlation coefficient. As the SNR correlation coefficient quantifies the statistical similarity between the end-to-end SNRs for global and local CSI, the proposed PPS with local CSI providing high SNR correlation can work most similarly to the BPS with global CSI. Assuming DF relays and Nakagami- $m$ fading channels, we present an exact and closed-form expression for the SNR correlation coefficient. In addition, we provide an exact and closed-form expression for the end-to-end outage probability of the PPS using local CSI so as to investigate an impact of the SNR correlation coefficient on the end-to-end outage probability of PPS and compare the end-to-end performances of BPS and PPS using the SNR correlation.

Main contributions of this paper are summarized as follows:

- The PPS scheme for dual-hop relaying systems is developed and generalized for multi-hop ones under Nakagami- $m$ fading channels.

- The correlation coefficient between the end-to-end SNRs for local CSI for a subset of hops and global CSI on a given path between a source and a destination is inceptively introduced to quantify a level of similarity between those for the local and global CSI.

- The exact SNR correlation coefficient for a given subset of hops on a given path is derived under Nakagami- $m$ fading channels, and its closed-form expression is provided.

- The exact and closed-form expression for the end-to-end outage probability of PPS with local CSI in Nakagami- $m$ fading channels is derived to evaluate the end-to-end performance of PPS based on the SNR correlation coefficient.

- Through numerical investigation, we show the relationship between the SNR correlation coefficient and the end-to-end outage probability of PPS and find certain channel conditions in order that the PPS with the highest SNR correlation coefficient achieves the similar performance to the BPS. 
The rest of this paper is organized as follows: related works to our research are covered in Section 2. In Section 3, we present the system model for multi-hop cooperative communications with parallel relays, BPS and PPS schemes, and their SNR distributions assuming independent Nakagami- $m$ fading channels. In Section 4, we introduce the correlation coefficient between the end-to-end SNRs for local and global CSI in order to find the best PPS and derive a closed-form expression for the SNR correlation coefficient. In Section 5, the end-to-end outage probability is analyzed to evaluate the end-to-end performance of the multi-hop cooperative system with the PPS using local CSI. In Section 6, we provide numerical results to verify the analysis of the SNR correlation coefficient and outage probability and show the impact of the SNR correlation coefficient on the end-to-end performance of PPS. Finally, in Section 7, we conclude the paper.

\section{Related Work}

For multi-hop transmissions, there are two typical relaying schemes: AF and DF relaying. In the AF relaying scheme, a relay amplifies a signal received from a previous node (or a source) and then forwards the amplified signal to a next node (or a destination). Because of the simple AF relaying operation, low complexity is required at the relay, but there is a noise propagation problem, which can induce a certain performance degradation. On the other hand, in the DF relaying scheme, a relay decodes and re-encodes a received signal from a previous node and then forwards the encoded signal to a next node. Because of the decoding operation in the DF relaying scheme, the noise propagation does not occur in multi-hop transmissions. However, DF relaying needs a relay with the higher complexity than AF relaying for its decoding and re-encoding operations.

To achieve spatial diversity in multi-hop relaying systems, the multi-hop cooperation techniques have been widely studied, where multiple cooperative nodes between a source and a destination are used as virtual antennas of the source or the destination. A simple but efficient multi-hop cooperation scheme is the BPS. In [11], the BPS schemes were proposed for dual-hop cooperative systems with AF relaying as well as DF relaying in order to achieve full spatial diversity. Also, their outage probability and diversity-multiplexing trade-off were analyzed for Rayleigh and Ricean fading channels. In [23,24], average bit error rate of the BPS was evaluated for multi-hop DF cooperative systems in Rayleigh and Nakagami- $m$ fading channels, respectively, where it was noted that more than two hops for each of every path were considered.

For green wireless communication, energy-efficient radio technologies have been presented in [12], where the issues on channel feedback information and CSI feedback overhead have been addressed to improve the energy efficiency. In [13], it was shown that the spectral efficiency and energy efficiency can be affected by channel feedback information in wireless multiple-input multiple-output transmission. In [14], the reduction in the CSI feedback overhead was studied to enhance the energy efficiency in downlink orthogonal frequency division multiple access. In [15], the relay and user selection scheme with one-bit CSI feedback was proposed for energy-efficient multiple-relay and multiple-user systems, and also exact expressions for outage probability and feedback requirement were provided to quantify the trade-off between complexity and performance. As mentioned previously, CSI feedback has been widely studied to achieve high energy efficiency in wireless communication.

The operation of the BPS is done by global CSI, and thus full CSI feedback is required. Hence, the CSI feedback for BPS can be limited from the viewpoint of energy efficiency for wireless communication systems. Therefore, in [16], the PPS was introduced in dual-hop AF relaying systems to utilize local CSI for path selection, and the statistical analysis of its end-to-end SNR was presented for Rayleigh fading channels. The PPS simply uses the CSI for one of two hops on each path. Thus, its end-to-end performance can be very limited. To improve the performance of the PPS, in [17], the efficient PPS was hence proposed for dual-hop AF relaying systems, and its outage probability was investigated in Rayleigh fading channels. In [17], it was also shown that the efficient PPS can provide the similar outage performance to the BPS under non-identical channel conditions in two-hop 
networks. In $[25,26]$, average bit error rate and outage probability of the efficient PPS were analyzed for dual-hop DF relaying systems in Rayleigh and Nakagami- $m$ fading channels.

The above-mentioned works assumed ideal CSI feedback, i.e., no feedback delay and no channel estimation error. However, in real wireless communication environments, there are the time delay between the CSI feedback and the data transmission as well as the channel estimation error at a receiver. Therefore, in $[27,28]$, outage probability, symbol error rate and bit error rate of the BPS and PPS with outdated channel estimates were respectively investigated for dual-hop AF relaying systems in Rayleigh fading channels. In [29], the outage probability of the efficient PPS with outdated CSI and channel estimation error was presented for dual-hop DF relaying systems in Rayleigh fading channels.

The study on efficient PPS has focused on only dual-hop cooperative systems so far. Hence, in this paper, we extend the dual-hop system model to the multi-hop (i.e., more than two hops) system model and focus on proposing a novel efficient PPS scheme for multi-hop DF relaying systems with limited CSI feedback, where ideal CSI feedback is assumed to show the best outage performance of the proposed efficient PPS and compare with the outage performance of the BPS. In addition, we consider Nakagami- $m$ fading channels to analyze its outage probability.

\section{System Model and SNR Distributions}

We consider a multi-hop parallel relay system (MRS), as shown in Figure 1 [23], where $K$ parallel independent paths are assumed to be available for data transmission from a source to a destination, and the $k$-th path between the source and the destination consists of $N_{k}$ serial hops with $N_{k}-1$ relays. In MRS, one of $K$ paths can be selected for data transmission to achieve spatial diversity according to the selection combining rule, i.e., only a single path with the best end-to-end link quality between the source and the destination can be chosen for transmission. The path selection is assumed to be performed by the source using CSI reported by the nodes. The relays operate in the half-duplex DF mode, where the half-duplex mode means that each relay is not allowed to transmit and receive the signal at the same time. Then, for orthogonal transmission, relay $n$ decodes a data signal received from the preceding node in the $n$-th time slot, and retransmits the decoded signal to the succeeding node during the $(n+1)$-th time slot. It is assumed that data communication is available only between adjacent nodes due to high path-loss and shadowing, and there are no CSI feedback delay and error.

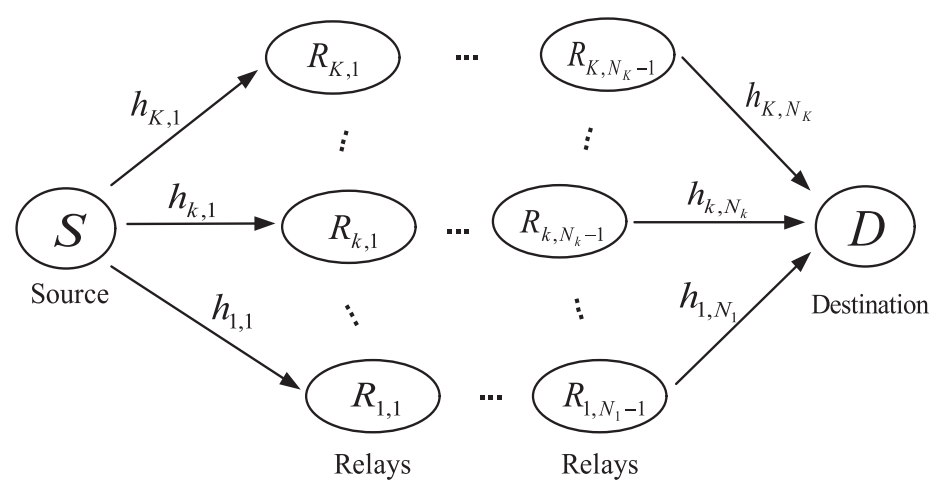

Figure 1. A multi-hop WSN with parallel relays from a source to a destination.

Let the channel coefficient for the $n$-th hop of the $k$-th path be denoted as $h_{k, n}$, and its magnitude be assumed to follow Nakagami- $m$ distribution with the integer parameter $m_{k, n}$ and the average power $\beta_{k, n}$, where it is noted that Nakagami- $m$ fading channels can generally represent line-of-sight as well as non-line-of-sight channel environments. It is also assumed that all the channels are independent. The received SNR for hop $n$ of path $k$ is then given as $\gamma_{k, n}=P_{k, n}\left|h_{k, n}\right|^{2} / \sigma_{k, n}^{2}$, where $P_{k, n}$ and $\sigma_{k, n}^{2}$ denote the transmit power and noise power, respectively.

The end-to-end SNR for DF relaying on path $k$ is defined as the weakest of the received SNRs for each of $N_{k}$ hops [23] and expressed as $q_{k} \triangleq \min _{n=1, \cdots, N_{k}}\left\{\gamma_{k, n}\right\}$, which also denotes the end-to-end SNR 
for global CSI, i.e, end-to-end perfect CSI. For the BPS, the path with the maximum end-to-end SNR among $K$ paths is chosen as follows: $k^{G}=\arg \max _{k=1, \cdots, K}\left\{q_{k}\right\}$. However, only local CSI (i.e., any subset of global CSI as a limited version) can be available at the source in practice because of limited CSI feedback, and then a path can be selected by the local CSI-based PPS as follows: $k^{L}=\arg \max _{k=1, \cdots, K}\left\{q_{k, l_{k}}\right\}$, where $l_{k}$ is the number of hops chosen for CSI feedback in path $k$, and $q_{k, l_{k}}=\min _{n=1, \cdots, l_{k}}\left\{\gamma_{k, n}\right\}$, which represents the end-to-end SNR for local CSI. For the simplicity of mathematical expressions, the subset of hops in path $k$ is set to $\left\{1, \cdots, l_{k}\right\}$, but it can be easily generalized to any subset of hops with size $l_{k}$.

Let $\rho_{k, n} \triangleq P_{k, n} \beta_{k, n} /\left(\sigma_{k, n}^{2} m_{k, n}\right)$. The probability density function (PDF) and the cumulative distribution function (CDF) of $\gamma_{k, n}$ are respectively given by

$$
\begin{aligned}
& f_{\gamma_{k, n}}(x)=\frac{1}{\Gamma\left(m_{k, n}\right)}\left(\frac{1}{\rho_{k, n}}\right)^{m_{k, n}} x^{m_{k, n}-1} e^{-\frac{x}{\rho_{k, n}}}, \\
& F_{\gamma_{k, n}}(x)=1-e^{-\frac{x}{\rho_{k, n}}} \sum_{i=0}^{m_{k, n}-1} \frac{1}{\Gamma(i+1)}\left(\frac{x}{\rho_{k, n}}\right)^{i},
\end{aligned}
$$

where $\Gamma(\cdot)$ is a gamma function [30]. Using Equation (2), the CDF of $q_{k, l_{k}}(x)$ is obtained as

$$
\begin{aligned}
F_{q_{k, l_{k}}}(x) & =1-\operatorname{Pr}\left\{\gamma_{k, 1}>x, \cdots, \gamma_{k, l_{k}}>x\right\} \\
& =1-e^{-\sum_{j=1}^{l_{k}} \frac{x}{\rho_{k, j}}}\left\{\sum_{i_{1}=0}^{m_{k, 1}-1} \cdots \sum_{i_{l_{k}}=0}^{m_{k} l_{k}-1} \prod_{j=1}^{l_{k}} \frac{1}{\Gamma\left(i_{j}+1\right)}\left(\frac{x}{\rho_{k, j}}\right)^{i_{j}}\right\} .
\end{aligned}
$$

Using Equation (3), the PDF of $q_{k, l_{k}}(x)$ is obtained as

$$
\begin{aligned}
f_{q_{k, l_{k}}}(x)= & \frac{d}{d x} F_{q_{k, l_{k}}}(x) \\
= & \sum_{i_{1}=0}^{m_{k, 1}-1} \cdots \sum_{i_{l_{k}}=0}^{m_{k, l_{k}}-1}\left\{\prod_{j=1}^{l_{k}} \frac{1}{\Gamma\left(i_{j}+1\right)}\left(\frac{1}{\rho_{k, j}}\right)^{i_{j}}\right\} \\
& \times\left\{\left(\sum_{j=1}^{l_{k}} \frac{1}{\rho_{k, j}}\right) x^{\sum_{j=1}^{l_{k}} i_{j}} e^{-\sum_{j=1}^{l_{k}} \frac{x}{\rho_{k, j}}}-\left(\sum_{j=1}^{l_{k}} i_{j}\right) \times x^{\left(\sum_{j=1}^{l_{k}} i_{j}\right)-1} e^{-\sum_{j=1}^{l_{k}} \frac{x}{\rho_{k, j}}}\right\} .
\end{aligned}
$$

\section{End-to-End SNR Correlation Analysis}

Based on the limited CSI feedback, $l_{k}$ 's for all paths are able to be decided, and in path $k$, an appropriate subset with size $l_{k}$ in the entire set of hops $\left\{1, \cdots, N_{k}\right\}$ can be determined for local CSI to provide the similar end-to-end SNR to global CSI (i.e., CSI for $N_{k}$ hops), which can enable the PPS using local CSI to be done like the BPS using global CSI. In this section, we analyze the correlation coefficient between $q_{k}$ and $q_{k, l_{k}}$ to find the best set of $l_{k}$ hops for local CSI. The correlation coefficient represents the quantity of the similarity between $q_{k}$ and $q_{k, l_{k}}$, and as it becomes larger, $q_{k}$ and $q_{k, l_{k}}$ become statistically more similar. For path $k$, the correlation coefficient between $q_{k}$ and $q_{k, l_{k}}$ is expressed as

$$
\eta_{k, l_{k}}=\frac{E\left[q_{k} q_{k, l_{k}}\right]-E\left[q_{k}\right] E\left[q_{k, l_{k}}\right]}{\sqrt{\left\{E\left[q_{k}^{2}\right]-\left(E\left[q_{k}\right]\right)^{2}\right\}\left\{E\left[q_{k, l_{k}}^{2}\right]-\left(E\left[q_{k, l_{k}}\right]\right)^{2}\right\}}},
$$

where $-1 \leq \eta_{k, l_{k}} \leq 1$, and $q_{k}$ and $q_{k, l_{k}}$ are highly correlated when $\eta_{k, l_{k}}$ approaches 1 .

Using Equation (4), the $t$-th moment of $f_{q_{k, l_{k}}}(x)$ is yielded as

$$
\begin{aligned}
E\left[q_{k, l_{k}}^{t}\right] & =\int_{0}^{\infty} x^{t} f_{q_{k, l_{k}}}(x) d x \\
& =t \sum_{i_{1}=0}^{m_{k, 1}-1} \cdots \sum_{i_{l_{k}}=0}^{m_{k, l_{k}}-1}\left\{\prod_{j=1}^{l_{k}} \frac{1}{\Gamma\left(i_{j}+1\right)}\left(\frac{1}{\rho_{k, j}}\right)^{i_{j}}\right\} \times \Gamma\left(t+\sum_{j=1}^{l_{k}} i_{j}\right)\left(\sum_{j=1}^{l_{k}} \frac{1}{\rho_{k, j}}\right)^{-t-\sum_{j=1}^{l_{k}} i_{j}} .
\end{aligned}
$$


By replacing $l_{k}$ with $N_{k}$ in Equation (6), $E\left[q_{k}^{t}\right]$ is also obtained. Then, $E\left[q_{k} q_{k, l_{k}}\right]$ is derived as

$$
\begin{aligned}
E\left[q_{k} q_{k, l_{k}}\right]= & \int_{0}^{\infty} \cdots \int_{0}^{\infty}\left[\min _{n=1, \cdots, N_{k}}\left\{x_{n}\right\}\right]\left[\min _{j=1, \cdots, l_{k}}\left\{x_{j}\right\}\right] f_{\gamma_{k, 1}}\left(x_{1}\right) \cdots f_{\gamma_{k, N_{k}}}\left(x_{N_{k}}\right) \\
& \times d x_{1} \cdots d x_{N_{k}} \\
= & \int_{0}^{\infty} \cdots \int_{0}^{\infty}\left[\min _{n=l_{k}+1, \cdots, N_{k}}\left\{x_{n}, y\right\}\right] y f_{\gamma_{k, l_{k}+1}}\left(x_{l_{k}+1}\right) \cdots f_{\gamma_{k, N_{k}}}\left(x_{N_{k}}\right) f_{q_{k, l_{k}}}(y) \\
& \times d x_{l_{k}+1} \cdots d x_{N_{k}} d y \\
= & \sum_{n=l_{k}+1}^{N_{k}} \int_{0}^{\infty} \int_{x_{n}}^{\infty} \cdots \int_{x_{n}}^{\infty} x_{n} y f_{q_{k, l_{k}}}(y) f_{\gamma_{k, l_{k}+1}}\left(x_{l_{k}+1}\right) \cdots f_{\gamma_{k, N_{k}}}\left(x_{N_{k}}\right) \\
& \times d y d x_{l_{k}+1} \cdots d x_{n-1} d x_{n+1} \cdots d x_{N_{k}} d x_{n} \\
& +\int_{0}^{\infty} \int_{y}^{\infty} \cdots \int_{y}^{\infty} y^{2} f_{\gamma_{k, l_{k}+1}}\left(x_{l_{k}+1}\right) \cdots f_{\gamma_{k, N_{k}}}\left(x_{N_{k}}\right) f_{q_{k, l_{k}}}(y) \\
& \times d x_{l_{k}+1} \cdots d x_{N_{k}} d y .
\end{aligned}
$$

To derive the first part of Equation (7), using Equations (1) and (4), we first have

$$
\begin{aligned}
& T_{1}=\int_{x_{n}}^{\infty} y f_{q_{k, l_{k}}}(y) d y \\
& =\sum_{i_{1}=0}^{m_{k, 1}-1} \cdots \sum_{i_{l_{k}}=0}^{m_{k, l_{k}}-1}\left(\prod_{j=1}^{l_{k}} \frac{\rho_{k, j}^{-i_{j}}}{\Gamma\left(i_{j}+1\right)}\right) \\
& \times\left\{e^{-\sum_{j=1}^{l_{k}} \frac{x_{n}}{\rho_{k, j}}} \sum_{u=0}^{1+\sum_{j=1}^{l_{k}} i_{j}} \frac{\Gamma\left(2+\sum_{j=1}^{l_{k}} i_{j}\right)}{\Gamma(u+1)}\left(\sum_{j=1}^{l_{k}} \frac{1}{\rho_{k, j}}\right)^{-1+u-\sum_{j=1}^{l_{k}} i_{j}} x_{n}^{u}\right. \\
& \left.-\left(\sum_{j=1}^{l_{k}} i_{j}\right) e^{-\sum_{j=1}^{l_{k}} \frac{x_{n}}{\rho_{k, j}}} \sum_{u=0}^{\sum_{j=1}^{l_{k}} i_{j}} \frac{\Gamma\left(1+\sum_{j=1}^{l_{k}} i_{j}\right)}{\Gamma(u+1)} \times\left(\sum_{j=1}^{l_{k}} \frac{1}{\rho_{k, j}}\right)^{-1+u-\sum_{j=1}^{l_{k}} i_{j}} x_{n}^{u}\right\}, \\
& T_{2}=\int_{x_{n}}^{\infty} \cdots \int_{x_{n}}^{\infty} \prod_{j=l_{k}+1, j \neq n}^{N_{k}} f_{\gamma_{k, j}}\left(x_{j}\right) d x_{j} \\
& =\sum_{i_{l_{k}+1}=0}^{m_{k, l_{k}+1}-1} \cdots \sum_{i_{n-1}=0}^{m_{k, n-1}-1} \sum_{i_{n+1}=0}^{m_{k, n+1}-1} \cdots \sum_{i_{N_{k}}=0}^{m_{k, N_{k}}-1}\left(\prod_{\substack{j=l_{k}+1, j \neq n}}^{\rho_{k}} \frac{\rho_{k, j}^{-i_{j}}}{\Gamma\left(i_{j}+1\right)}\right) \times e^{-\sum_{j=l_{k}+1, j \neq n}^{N_{k}} \frac{x}{n}_{p_{k, j}}} x_{n}^{\sum_{j=k_{k}+1, j \neq n}^{N_{k} i_{j}}} .
\end{aligned}
$$

Multiplying Equations (8) and (9), then we obtain the first part of Equation (7) as follows:

$$
\begin{aligned}
& \sum_{n=l_{k}+1}^{N_{k}} \int_{0}^{\infty} x_{n} T_{1} T_{2} f_{\gamma_{k, n}}\left(x_{n}\right) d x_{n} \\
& =\sum_{n=l_{k}+1}^{N_{k}} \sum_{i_{l_{1}}=0}^{m_{k, 1}-1} \cdots \sum_{i_{k-1}=0}^{m_{k, n-1}-1} \sum_{i_{n+1}=0}^{m_{k, n+1}-1} \cdots \sum_{i_{N_{k}}=0}^{m_{k, N_{k}}-1} \frac{\rho_{k, n}^{-m_{k, n}}}{\Gamma\left(m_{k, n}\right)} \\
& \quad \times\left(\prod_{j=1, j \neq n}^{N_{k}} \frac{\rho_{k_{j}}}{\Gamma\left(i_{j}+1\right)}\right)\left\{\Gamma\left(\omega_{k, n}\right)\left(\sum_{j=1}^{N_{k}} \frac{1}{\rho_{k, j}}\right)^{-\omega_{k, n}}+\sum_{u=0}^{\sum_{j=1}^{l_{k}} i_{j}} \frac{\Gamma\left(1+\sum_{j=1}^{l_{k}} i_{j}\right)}{\Gamma(u+1)}\left(\sum_{j=1}^{l_{k}} \frac{1}{\rho_{k, j}}\right)^{-1+u-\sum_{j=1}^{l_{k}} i_{j}}\right. \\
& \left.\quad \times \Gamma\left(\lambda_{k, n}\right)\left(\sum_{j=1}^{N_{k}} \frac{1}{\rho_{k, j}}\right)^{-\lambda_{k, n}}\right\}
\end{aligned}
$$


where $\omega_{k, n}=m_{k, n}+2+\sum_{j=1, j \neq n}^{N_{k}} i_{j}$, and $\lambda_{k, n}=u+m_{k, n}+1+\sum_{j=l_{k}+1, j \neq n}^{N_{k}} i_{j}$. Using Equations (1) and (4), we derive the other part of Equation (7) as follows:

$$
\begin{aligned}
& \int_{0}^{\infty} \int_{y}^{\infty} \cdots \int_{y}^{\infty} y^{2} f_{\gamma_{k, l_{k}+1}}\left(x_{l_{k}+1}\right) \cdots f_{\gamma_{k, N_{k}}}\left(x_{N_{k}}\right) f_{q_{k, l_{k}}}(y) d x_{l_{k}+1} \cdots d x_{N_{k}} d y \\
& =\sum_{i_{1}=0}^{m_{k, 1}-1} \cdots \sum_{i_{N_{k}}=0}^{m_{k, N_{k}}-1}\left(\prod_{j=1}^{N_{k}} \frac{\rho_{k, j}^{-i_{j}}}{\Gamma\left(i_{j}+1\right)}\right)\left\{\left(\sum_{j=1}^{l_{k}} \frac{1}{\rho_{k, j}}\right) \int_{0}^{\infty} y^{2+\sum_{j=1}^{N_{k}} i_{j}} e^{-\sum_{j=1}^{N_{k}} \frac{y}{\rho_{k, j}}} d y\right. \\
& \left.-\left(\sum_{j=1}^{l_{k}} i_{j}\right) \int_{0}^{\infty} y^{1+\sum_{j=1}^{N_{k}} i_{j}} e^{-\sum_{j=1}^{N_{k}} \frac{y}{\rho_{k, j}}} d y\right\} \\
& =\sum_{i_{1}=0}^{m_{k, 1}-1} \cdots \sum_{i_{N_{k}}=0}^{m_{k, N_{k}}-1}\left(\prod_{j=1}^{N_{k}} \frac{\rho_{k, j}^{-i_{j}}}{\Gamma\left(i_{j}+1\right)}\right)\left\{\left(2+\sum_{j=1}^{N_{k}} i_{j}\right)\left(\sum_{j=1}^{l_{k}} \frac{1}{\rho_{k, j}}\right)\left(\sum_{j=1}^{N_{k}} \frac{1}{\rho_{k, j}}\right)^{-1}\right. \\
& \left.-\sum_{j=1}^{l_{k}} i_{j}\right\} \Gamma\left(2+\sum_{j=1}^{N_{k}} i_{j}\right)\left(\sum_{j=1}^{N_{k}} \frac{1}{\rho_{k, j}}\right)^{-2-\sum_{j=1}^{N_{k}} i_{j}} .
\end{aligned}
$$

Finally, $E\left[q_{k} q_{k, l_{k}}\right]$ is yielded by the sum of Equations (10) and (11) as follows:

$$
\begin{aligned}
& E\left[q_{k} q_{k, l_{k}}\right]=\left[\sum _ { n = l _ { k } + 1 } ^ { N _ { k } } \sum _ { i _ { 1 } = 0 } ^ { m _ { k , 1 } - 1 } \cdots \sum _ { i _ { n - 1 } = 0 } ^ { m _ { k , n - 1 } - 1 } \sum _ { i _ { n + 1 } = 0 } ^ { m _ { k , n + 1 } - 1 } \cdots \sum _ { i _ { N _ { k } } = 0 } ^ { m _ { k , N _ { k } } - 1 } \frac { \rho _ { k , n } ^ { - m _ { k , n } } } { \Gamma ( m _ { k , n } ) } ( \prod _ { j = 1 , j \neq n } ^ { N _ { k } } \frac { \rho _ { k _ { k j } } ^ { - i _ { j } } } { \Gamma ( i _ { j } + 1 ) } ) \left\{\Gamma\left(\omega_{k, n}\right)\right.\right. \\
& \left.\left.\times\left(\sum_{j=1}^{N_{k}} \frac{1}{\rho_{k, j}}\right)^{-\omega_{k, n}}+\sum_{u=0}^{\sum_{j=1}^{l_{k}} i_{j}} \frac{\Gamma\left(1+\sum_{j=1}^{l_{k}} i_{j}\right)}{\Gamma(u+1)}\left(\sum_{j=1}^{l_{k}} \frac{1}{\rho_{k, j}}\right)^{-1+u-\sum_{j=1}^{l_{k}} i_{j}} \Gamma\left(\lambda_{k, n}\right)\left(\sum_{j=1}^{N_{k}} \frac{1}{\rho_{k, j}}\right)^{-\lambda_{k, n}}\right\}\right] \\
& +\left[\sum_{i_{1}=0}^{m_{k, 1}-1} \cdots \sum_{i_{N_{k}}=0}^{m_{k, N_{k}}-1}\left(\prod_{j=1}^{N_{k}} \frac{\rho_{k_{k j}}^{-i_{j}}}{\Gamma\left(i_{j}+1\right)}\right)\left\{\left(2+\sum_{j=1}^{N_{k}} i_{j}\right)\left(\sum_{j=1}^{l_{k}} \frac{1}{\rho_{k, j}}\right)\left(\sum_{j=1}^{N_{k}} \frac{1}{\rho_{k, j}}\right)^{-1}-\sum_{j=1}^{l_{k}} i_{j}\right\}\right. \\
& \left.\times \Gamma\left(2+\sum_{j=1}^{N_{k}} i_{j}\right)\left(\sum_{j=1}^{N_{k}} \frac{1}{\rho_{k, j}}\right)^{-2-\sum_{j=1}^{N_{k}} i_{j}}\right] \text {. }
\end{aligned}
$$

Therefore, using $E\left[q_{k} q_{k, l_{k}}\right]$ in Equation (12) and $E\left[q_{k}\right], E\left[q_{k, l_{k}}\right], E\left[q_{k}^{2}\right]$ and $E\left[q_{k, l_{k}}^{2}\right]$ given from Equation (6), a closed-form expression for the correlation coefficient $\eta_{k, l_{k}}$ in Equation (5) is obtained.

As mentioned in Section 3, it is assumed that the subset of hops for CSI feedback in path $k$ is set to $\left\{1, \cdots, l_{k}\right\}$ for the simplification of the mathematical expressions, but it can be easily extended to any subset of hops with size $l_{k}$, denoted by $\left\{\delta_{1}, \cdots, \delta_{l_{k}}\right\}$, where $\delta$ denotes the hop index. Then, the possible subsets $\left\{\delta_{1}, \cdots, \delta_{l_{k}}\right\}$ are respectively used to calculate $\eta_{k, l_{k}}$ using its closed-form expression, and the best subset of hops with size $l_{k}$ in path $k$ is found to maximize $\eta_{k, l_{k}}$, where it implies that $q_{k, l_{k}}$ for the best subset provides the best similarity to $q_{k}$. Thus, the PPS using only instantaneous CSI for the best subset of hops in each of paths can work most analogously to the BPS using global instantaneous CSI for every path. It is noted that although the closed-form expression for $\eta_{k, l_{k}}$ may be complicated, only the Nakagami parameters $m_{k, n}$ 's and the average SNRs $\rho_{k, n}$ 's are required to calculate $\eta_{k, l_{k}}$, where all the values of the parameters may slowly change over time. Furthermore, it is noted that the PPS based on the SNR correlation can be also employed in AF relaying systems since the end-to-end SNR of AF relaying is tightly upper-bounded by that of DF relaying [31].

For Rayleigh fading channels (i.e., $m_{k, n}=1$ for all $k$ and $n$ ), using Equations (6) and (12), the closed-form expression for $\eta_{k, l_{k}}$ in Equation (5) can be simplified as follows:

$$
\begin{aligned}
\eta_{k, l_{k}}= & {\left[\left(\sum_{j=l_{k}+1}^{N_{k}} \frac{1}{\rho_{k, j}}\right)\left\{2\left(\sum_{j=1}^{N_{k}} \frac{1}{\rho_{k, j}}\right)^{-3}+\left(\sum_{j=1}^{l_{k}} \frac{1}{\rho_{k, j}}\right)^{-1}\left(\sum_{j=1}^{N_{k}} \frac{1}{\rho_{k, j}}\right)^{-2}\right\}+2\left(\sum_{j=1}^{l_{k}} \frac{1}{\rho_{k, j}}\right)\left(\sum_{j=1}^{N_{k}} \frac{1}{\rho_{k, j}}\right)^{-3}\right.} \\
& \left.-\left(\sum_{j=1}^{l_{k}} \frac{1}{\rho_{k, j}}\right)^{-1}\left(\sum_{j=1}^{N_{k}} \frac{1}{\rho_{k, j}}\right)^{-1}\right]\left(\sum_{j=1}^{l_{k}} \frac{1}{\rho_{k, j}}\right)\left(\sum_{j=1}^{N_{k}} \frac{1}{\rho_{k, j}}\right)\left(\sum_{j=1}^{l_{k}} \frac{1}{\rho_{k, j}}\right)\left(\sum_{j=1}^{N_{k}} \frac{1}{\rho_{k, j}}\right)^{-1}
\end{aligned}
$$

where it is noted that the correlation coefficient $\eta_{k, l_{k}}$ becomes larger as $\rho_{k, j}$ 's for $j=1, \cdots, l_{k}$ decrease. However, for Nakagami- $m$ fading channels, $\eta_{k, l_{k}}$ is affected by $\rho_{k, n}$ 's as well as $m_{k, n}$ 's, as seen in Equations (6) and (12). 


\section{Outage Probability Analysis}

In this paper, the outage probability is considered to be a performance metric to evaluate the end-to-end performance of the MRS with the PPS using local CSI and investigate the impact of the SNR correlation coefficient $\eta_{k, l_{k}}$ on the end-to-end performance. It is noted that the SNR correlation coefficient may also similarly affect other performance metrics such as bit/symbol error rate and ergodic capacity that are dependent upon a minimum SNR of hops for each path, where a minimum SNR for each path means $q_{k}$ in this paper.

Let the outage probability be defined as the probability that the end-to-end data rate falls below the target data rate $R_{t}$, where the end-to-end data rate for path $k$ is given by $\frac{1}{N_{k}} \log _{2}\left(1+q_{k}\right)$ as in [32]. Then, the outage probability of the MRS with the path selection is expressed as

$$
P_{\text {out }}=\sum_{k=1}^{K} \operatorname{Pr}\left\{\frac{1}{N_{\zeta}} \log _{2}\left(1+q_{\zeta}\right)<R_{t}, \zeta=k\right\}
$$

where $\zeta \in\left\{k^{G}, k^{L}\right\}$. In this section, we derive the outage probability only for the PPS using local CSI (i.e., $k^{L}$ ) since the outage probability for the BPS using global CSI (i.e., $k^{G}$ ) is easily obtained by replacing $l_{k}$ with $N_{k}$. From Equation (14), the outage probability for the local CSI-based PPS is derived as

$$
\begin{aligned}
P_{\text {out }}= & \sum_{k=1}^{K} \operatorname{Pr}\left\{q_{k}<z_{k}, q_{k, l_{k}}>q_{1, l_{1}}, \cdots, q_{k, l_{k}}>q_{k-1, l_{k-1}}, q_{k, l_{k}}>q_{k+1, l_{k+1}}, \cdots, q_{k, l_{k}}>q_{K, l_{K}}\right\} \\
= & \sum_{k=1}^{K}\left[\operatorname{Pr}\left\{q_{k, l_{k}}>q_{1, l_{1}}, \cdots, q_{k, l_{k}}>q_{k-1, l_{k-1}}, q_{k, l_{k}}>q_{k+1, l_{k+1}}, \cdots, q_{k, l_{k}}>q_{K, l_{K}}\right\}\right. \\
& \left.-\operatorname{Pr}\left\{q_{k}>z_{k}, q_{k, l_{k}}>q_{1, l_{1}}, \cdots, q_{k, l_{k}}>q_{k-1, l_{k-1}}, q_{k, l_{k}}>q_{k+1, l_{k+1}}, \cdots, q_{k, l_{k}}>q_{K, l_{K}}\right\}\right] \\
= & 1-\sum_{k=1}^{K}\left[\prod_{j=l_{k}+1}^{N_{k}} \operatorname{Pr}\left\{\gamma_{k, j}>z_{k}\right\}\right] \\
& \times \operatorname{Pr}\left\{q_{k, l_{k}}>z_{k}, q_{k, l_{k}}>q_{1, l_{1}}, \cdots, q_{k, l_{k}}>q_{k-1, l_{k-1}}, q_{k, l_{k}}>q_{k+1, l_{k+1}}, \cdots, q_{k, l_{k}}>q_{K, l_{K}}\right\} \\
= & 1-\sum_{k=1}^{K}\left\{\prod_{j=l_{k}+1}^{N_{k}}\left(1-F_{\gamma_{k, j}}\left(z_{k}\right)\right)\right\} \\
& \times \int_{z_{k}}^{\infty} \int_{0}^{y_{k}} \cdots \int_{0}^{y_{k}} f_{q_{1, l_{1}}}\left(y_{1}\right) \cdots f_{q_{K, l_{K}}}\left(y_{K}\right) d y_{1} \cdots d y_{k-1} d y_{k+1} \cdots d y_{K} d y_{k},
\end{aligned}
$$

where $z_{\zeta}=2^{N_{\zeta} R_{t}}-1$. In Equation (15), using Equation (2), we first obtain

$$
\prod_{j=l_{k}+1}^{N_{k}}\left(1-F_{\gamma_{k, j}}\left(z_{k}\right)\right)=\left\{\prod_{j=l_{k}+1}^{N_{k}}\left(e^{-\frac{z_{k}}{\rho_{k, j}}} \sum_{i=0}^{m_{k, j}-1} \frac{1}{\Gamma(i+1)}\left(\frac{z_{k}}{\rho_{k, j}}\right)^{i}\right)\right\} .
$$

Then, using Equation (4), we derive the integral term in Equation (15) as follows:

$$
\begin{aligned}
& \int_{z_{k}}^{\infty} \int_{0}^{y_{k}} \cdots \int_{0}^{y_{k}} f_{q_{1, l_{1}}}\left(y_{1}\right) \cdots f_{q_{K, l_{K}}}\left(y_{K}\right) d y_{1} \cdots d y_{k-1} d y_{k+1} \cdots d y_{K} d y_{k} \\
& =\int_{z_{k}}^{\infty}\left[1+\sum_{s=1}^{K-1}(-1)^{s} \sum_{u_{1}<\cdots<u_{s}} \sum_{i_{u_{1}, 1}=0}^{m_{u_{1}, 1}-1} \cdots \sum_{i_{u_{1}, u_{1}}=0}^{m_{u_{1}, u_{u_{1}}}-1} \cdots \sum_{i_{u_{s}, 1}=0}^{m_{u_{s}, 1}-1} \cdots \sum_{i_{u_{s}, u_{s}}=0}^{m_{u_{s}, l_{u_{s}}-1}=0}\left(\prod_{\alpha=1}^{s} \prod_{j=1}^{h_{u_{\alpha}}} \frac{\rho_{u_{u, j}}^{-i_{u_{\alpha}, j}}}{\Gamma\left(i_{u_{u}, j}+1\right)}\right)\right.
\end{aligned}
$$

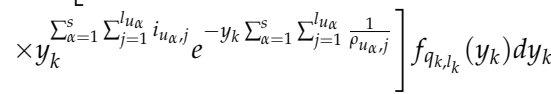

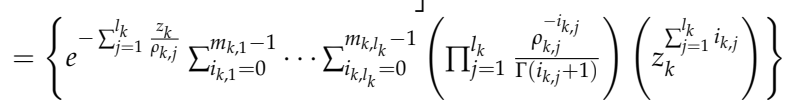

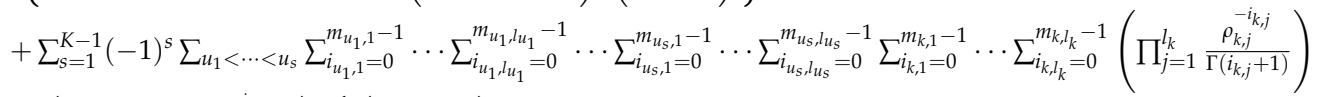

$$
\begin{aligned}
& \times\left(\prod_{\alpha=1}^{s} \prod_{j=1}^{l_{k}} \frac{\rho_{u_{\alpha}}^{-i_{u_{\alpha}, j}}}{\Gamma\left(i_{u_{\alpha}, j}+1\right)}\right)\left\{\left(\sum_{j=1}^{l_{k}} \frac{1}{\rho_{k, j}}\right) \Omega_{k, s}^{-\Lambda_{k, s}-1} \Gamma\left(\Lambda_{k, s}+1, \Omega_{k, s} z_{k}\right)\right. \\
& \left.-\left(\sum_{j=1}^{l_{k}} i_{k, j}\right) \Omega_{k, s}^{-\Lambda_{k, s}} \Gamma\left(\Lambda_{k, s}, \Omega_{k, s} z_{k}\right)\right\} \text {, }
\end{aligned}
$$


where $u_{1}<\cdots<u_{s}$ denote all the possible subsets with size $s$ in the set $\{1, \cdots, k-1, k+1, \cdots, K\}$, and $\Gamma(\cdot, \cdot)$ is an incomplete gamma function. Furthermore, $\Omega_{k, s}=\sum_{j=1}^{l_{k}}\left(1 / \rho_{k, j}\right)+\sum_{\alpha=1}^{s} \sum_{j=1}^{l_{u_{\alpha}}}\left(1 / \rho_{u_{\alpha}, j}\right)$, and $\Lambda_{k, s}=\sum_{j=1}^{l_{k}} i_{k, j}+\sum_{\alpha=1}^{s} \sum_{j=1}^{l_{u_{\alpha}}} i_{u_{\alpha}, j}$. Substituting Equations (16) and (17) into Equation (15), a closed-form expression for $P_{\text {out }}$ with the local CSI-based PPS is finally obtained as

$$
\begin{aligned}
& P_{\text {out }}=1-\sum_{k=1}^{K}\left\{\prod_{j=l_{k}+1}^{N_{k}}\left(e^{-\frac{z_{k}}{\rho_{k, j}}} \sum_{i=0}^{m_{k, j}-1} \frac{1}{\Gamma(i+1)}\left(\frac{z_{k}}{\rho_{k, j}}\right)^{i}\right)\right\}\left[\left\{e^{-\sum_{j=1}^{l_{k}} \frac{z_{k}}{\rho_{k, j}}} \sum_{i_{k, 1}=0}^{m_{k, 1}-1} \cdots \sum_{i_{k, l_{k}}=0}^{m_{k, l_{k}}-1}\left(z_{k}^{\sum_{j=1}^{l_{k}} i_{k, j}}\right)\right.\right. \\
& \left.\times\left(\prod_{j=1}^{l_{k}} \frac{\rho_{k, j}^{-i_{k, j}}}{\Gamma\left(i_{k, j}+1\right)}\right)\right\}+\sum_{s=1}^{K-1}(-1)^{s} \sum_{u_{1}<\cdots<u_{s}} \sum_{i_{u_{1}, 1}=0}^{m_{u_{1}, 1}-1} \cdots \sum_{i_{u_{1}, u_{1}}=0}^{m_{u_{1}, u_{1}}-1} \cdots \sum_{i_{u_{s}, 1}=0}^{m_{u_{s}, 1}-1} \cdots \sum_{i_{u_{s}, u_{s}}=0}^{m_{u_{s}, l_{s}}-1} \sum_{i_{k, 1}=0}^{m_{k, 1}-1} \cdots \\
& \sum_{i_{k, l_{k}}=0}^{m_{k, l_{k}}-1}\left(\prod_{j=1}^{l_{k}} \frac{\rho_{k_{j}, j}^{-i_{k}}}{\Gamma\left(i_{k, j}+1\right)}\right)\left(\prod_{\alpha=1}^{s} \prod_{j=1}^{l_{u_{\alpha}}} \frac{\rho_{u_{\alpha}, j}^{-i_{u_{\alpha}, j}}}{\Gamma\left(i_{u_{\alpha, j}}+1\right)}\right)\left\{\left(\sum_{j=1}^{l_{k}} \frac{1}{\rho_{k, j}}\right) \Omega_{k, s}^{-\Lambda_{k, s}-1} \Gamma\left(\Lambda_{k, s}+1, \Omega_{k, s} z_{k}\right)\right. \\
& \left.\left.-\left(\sum_{j=1}^{l_{k}} i_{k, j}\right) \Omega_{k, s}^{-\Lambda_{k, s}} \Gamma\left(\Lambda_{k, s}, \Omega_{k, s} z_{k}\right)\right\}\right] \text {. }
\end{aligned}
$$

When Rayleigh fading channels are assumed (i.e., $m_{k, n}=1$ for all $k$ and $n$ ), the closed-form expression for $P_{\text {out }}$ in Equation (18) can be shortened as

$$
P_{\text {out }}=1-\sum_{k=1}^{K} e^{-\sum_{j=l_{k}+1}^{N_{k}} \frac{z_{k}}{\rho_{k, j}}}\left\{e^{-\sum_{j=1}^{l_{k}} \frac{z_{k}}{\rho_{k, j}}}+\sum_{s=1}^{K-1}(-1)^{S} \sum_{u_{1}<\cdots<u_{s}}\left(\sum_{j=1}^{l_{k}} \frac{1}{\rho_{k, j}}\right) \Omega_{k, s}^{-1} e^{-\Omega_{k, s} z_{k}}\right\} .
$$

\section{Numerical Results}

For simulation, suppose $\beta_{k, 1}=1, \beta_{k, 2}=2 C_{p}$ and $\beta_{k, 3}=4 C_{p}$ for all $k$, where $C_{p} \geq 1$ denotes a proportional factor to adjust the difference between the average channel powers for hops, and $S_{k}$ represents the set of the hop indexes for local CSI reported for the PPS. In the following figures that show the numerical results of the outage probability, global CSI denotes the BPS using global CSI. In all the figures, the simulation results are obtained by Monte-Carlo simulations, in which $10^{6}$ channel realizations for each hop are generated from the Nakagami distribution. In Figures 2-5, the simulation results are indicated by only symbols (e.g., square, circle, triangle, etc.) without lines, while the analytical results are indicated by only solid lines.

Figures 2 and 3 show the SNR correlation coefficient $\eta_{k, l_{k}}$ and its corresponding outage probability $P_{\text {out }}$ for various $C_{p}$ with different local CSI sets when $m_{k, n}=1,2$ and $P_{k, n} / \sigma_{k, n}^{2}=22 \mathrm{~dB}, 16 \mathrm{~dB}$ for all $k$ and $n$, respectively. In addition, $N_{k}=3$ and $l_{k}=1,2$ for all $k$ (i.e., one and two of three hops for local CSI, respectively), $K=2$, and $R_{t}=1 \mathrm{bps} / \mathrm{Hz}$. The figures illustrate that $S_{k}=\{1\},\{1,2\}$ provide the largest $\eta_{k, l_{k}}$ for $l_{k}=1,2$, respectively, and their $\eta_{k, l_{k}}$ 's increase with $C_{p}$, which means that $\eta_{k, l_{k}}$ is dominated by small $\beta_{k, n}$, and rises as the gap between $\beta_{k, n}$ 's becomes larger. Remarkably, $\eta_{k, l_{k}}$ 's for $S_{k}=\{1\},\{1,2\}$ significantly increase with $m_{k, n}$, and become closer to 1 for high $C_{p}$. The figures also demonstrate that $P_{\text {out }}$ becomes better as $\eta_{k, l_{k}}$ goes up, and $P_{\text {out }}$ for the PPS with $l_{k}=2$ and $S_{k}=\{1,2\}$ for all $k$ is extremely similar to that for the BPS with global CSI when $m_{k, n}=2$ and $C_{p}$ is high. Even for $l_{k}=1$ and $S_{k}=\{1\}$, the analogous outage performance to global CSI is achieved for high $C_{p}$. In Figures $2 \mathrm{a}$ and $3 \mathrm{a}$, the analytic results of the SNR correlation coefficient and the outage probability, obtained by (13) and (19), respectively, perfectly match the corresponding simulation results. In Figures $2 b$ and $3 b$, the analytic results of the SNR correlation coefficient and the outage probability, obtained by (5) and (18), respectively, also well match their simulation results.

Letting $P_{t} / \sigma^{2} \triangleq P_{k, n} / \sigma_{k, n}^{2}$ for all $k$ and $n$, Figure 4 shows the outage probability for various transmit SNR $P_{t} / \sigma^{2}$ with $K=2,3$ and $R_{t}=1 \mathrm{bps} / \mathrm{Hz}$ when $m_{k, 1}=1, m_{k, 2}=m_{k, 3}=2,3, N_{k}=3$ and $l_{k}=1$ for all $k$. In addition, $\beta_{k, 1}=1, \beta_{k, 2}=4$, and $\beta_{k, 3}=8$ for all $k$. Then, for $l_{k}=1$ and $S_{k}=\{1\}$, $\eta_{k, 1}=0.866,0.9132$ with $m_{k, 1}=1$ and $m_{k, 2}=m_{k, 3}=2,3$, respectively, which means that when the smallest $\beta_{k, n}$ in path $k$ has relatively small $m_{k, n}$, its $\eta_{k, l_{k}}$ considerably increases. It is noted that $\eta_{k, 1}$ 's for all $k$ are the same because the channel conditions for every path are assumed to be identical. From that observation, the figure indicates that when $K=3, P_{\text {out }}$ for the PPS with $l_{k}=1$ and $S_{k}=\{1\}$ becomes 
extremely closer to that for the BPS with global CSI as $m_{k, 2}$ and $m_{k, 3}$ increase to 3 (i.e., $\eta_{k, 1}$ increases), where it is noted that even though only CSI for one of three hops is used for the PPS, it achieves the similar outage performance to the BPS with global CSI. It is also observed that as $K$ rises, better $\eta_{k, l_{k}}$ is required to attain the similar outage performance to the BPS. In addition, as the transmit SNR $P_{t} / \sigma^{2}$ decreases, $P_{\text {out }}$ for $l_{k}=1$ and $S_{k}=\{1\}$ is extremely closer to that for global CSI.

Figure 5 shows the outage probability for various transmit SNR $P_{t} / \sigma^{2}$ with $K=2,3$ and $R_{t}=$ $1,2 \mathrm{bps} / \mathrm{Hz}$ when $m_{k, n}=2,3, N_{k}=3$ and $l_{k}=2$ for all $k$ and $n$. In addition, $\beta_{k, 1}=1, \beta_{k, 2}=4$, and $\beta_{k, 3}=8$ for all $k$. Then, for $l_{k}=2$ and $n=\{1,2\}, \eta_{k, 2}=0.975,0.992$ with $m_{k, n}=2,3$ for all $n$, respectively. Analogous to the observation in Figure 4 , the PPS with higher $\eta_{k, 2}$ provides the similar outage performance to the BPS, but a small performance gap between the PPS and the BPS is observed for high transmit SNR since the BPS achieves more spatial diversity as all $m_{k, n}$ 's increase to 3. Nevertheless, it is noteworthy that the outage performance of the PPS using local CSI with $l_{k}=2$ and $n=\{1,2\}$ is extremely closer to that of the BPS using global CSI for low and medium transmit SNR regimes.

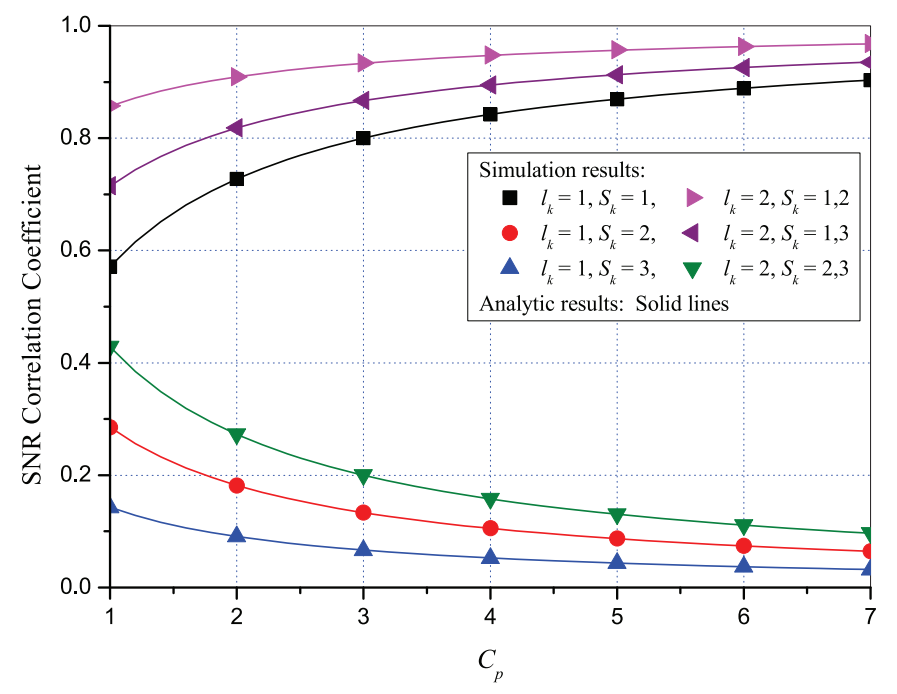

(a)

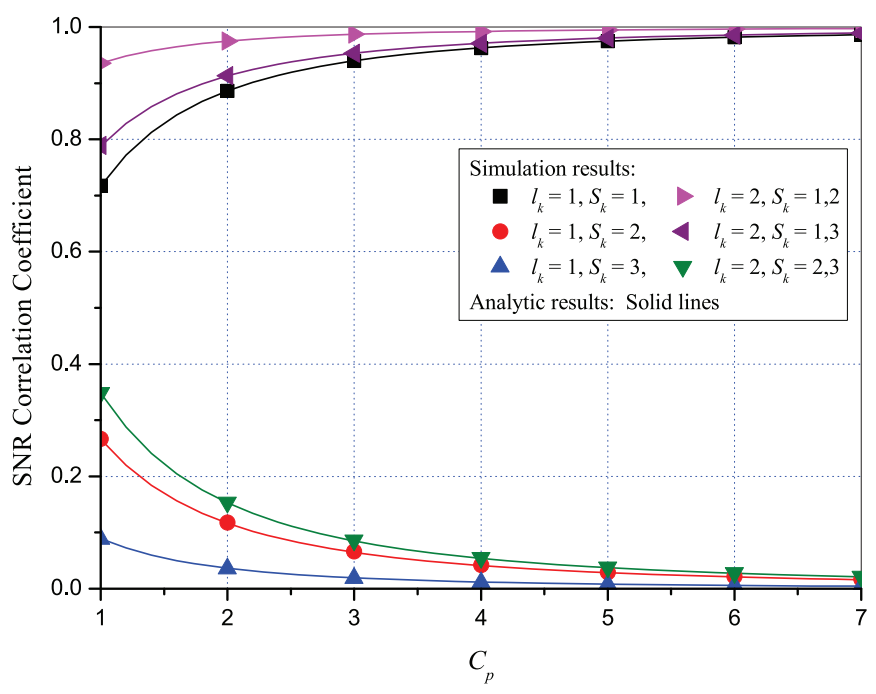

(b)

Figure 2. SNR correlation coefficient for $N_{k}=3$ for all $k$ when $K=2$ and $R_{t}=1 \mathrm{bps} / \mathrm{Hz}$ : (a) $P_{k, n} / \sigma_{k, n}^{2}=22 \mathrm{~dB}$ and $m_{k, n}=1$ for all $k$ and $n$; (b) $P_{k, n} / \sigma_{k, n}^{2}=16 \mathrm{~dB}$ and $m_{k, n}=2$ for all $k$ and $n$. 


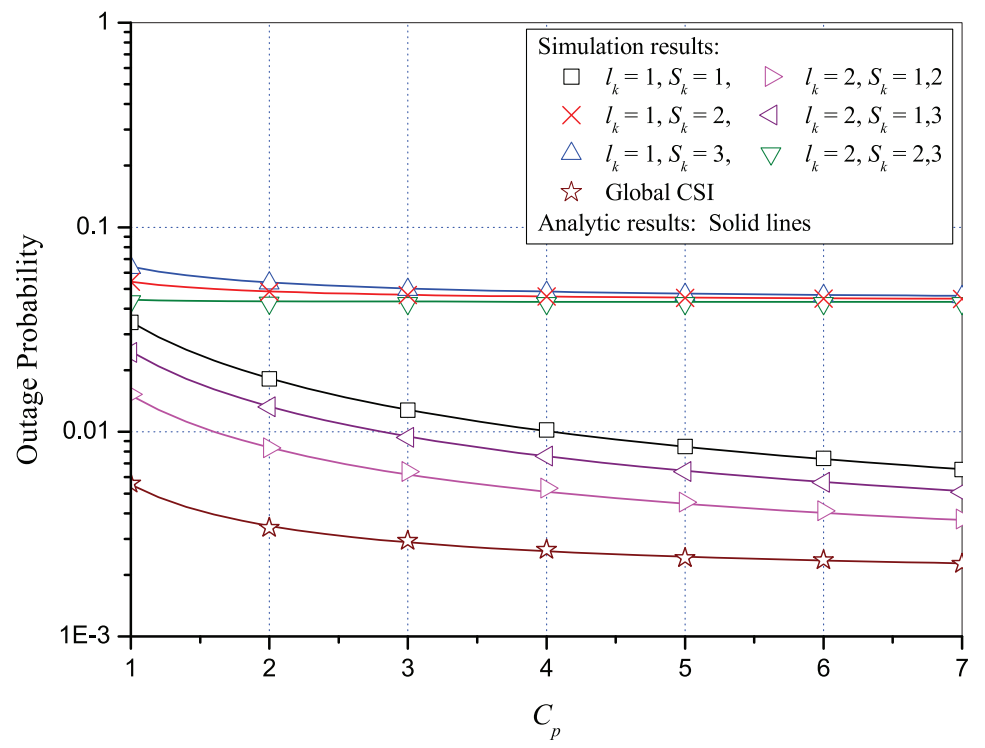

(a)

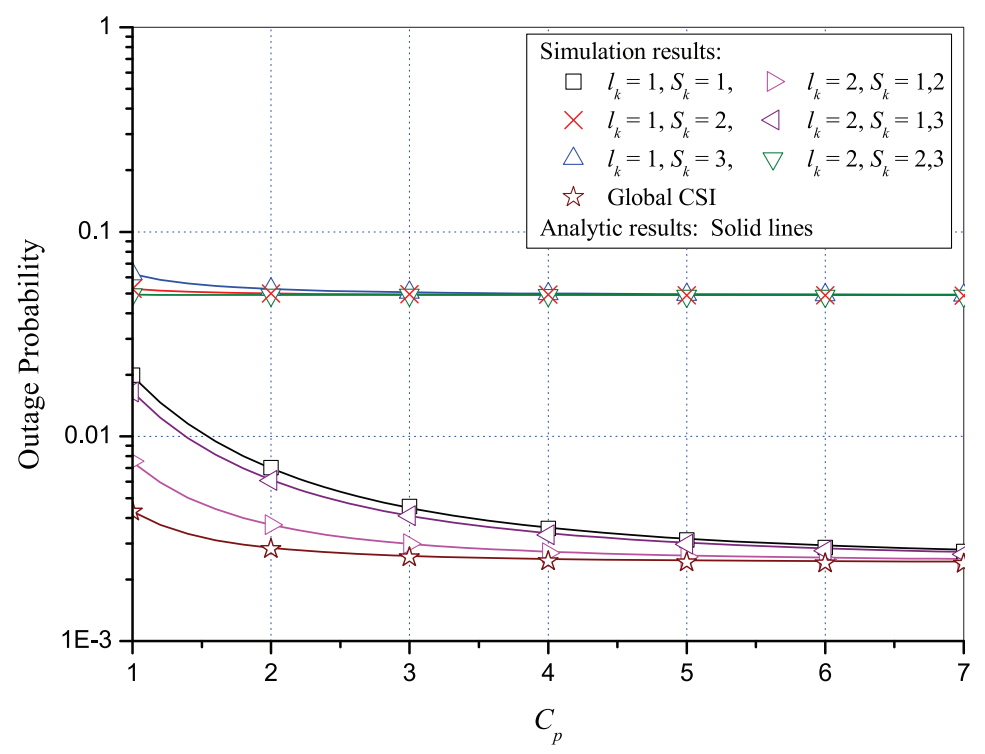

(b)

Figure 3. Outage probability for $N_{k}=3$ for all $k$ when $K=2$ and $R_{t}=1 \mathrm{bps} / \mathrm{Hz}$ : (a) $P_{k, n} / \sigma_{k, n}^{2}=22 \mathrm{~dB}$ and $m_{k, n}=1$ for all $k$ and $n ;(\mathbf{b}) P_{k, n} / \sigma_{k, n}^{2}=16 \mathrm{~dB}$ and $m_{k, n}=2$ for all $k$ and $n$. 


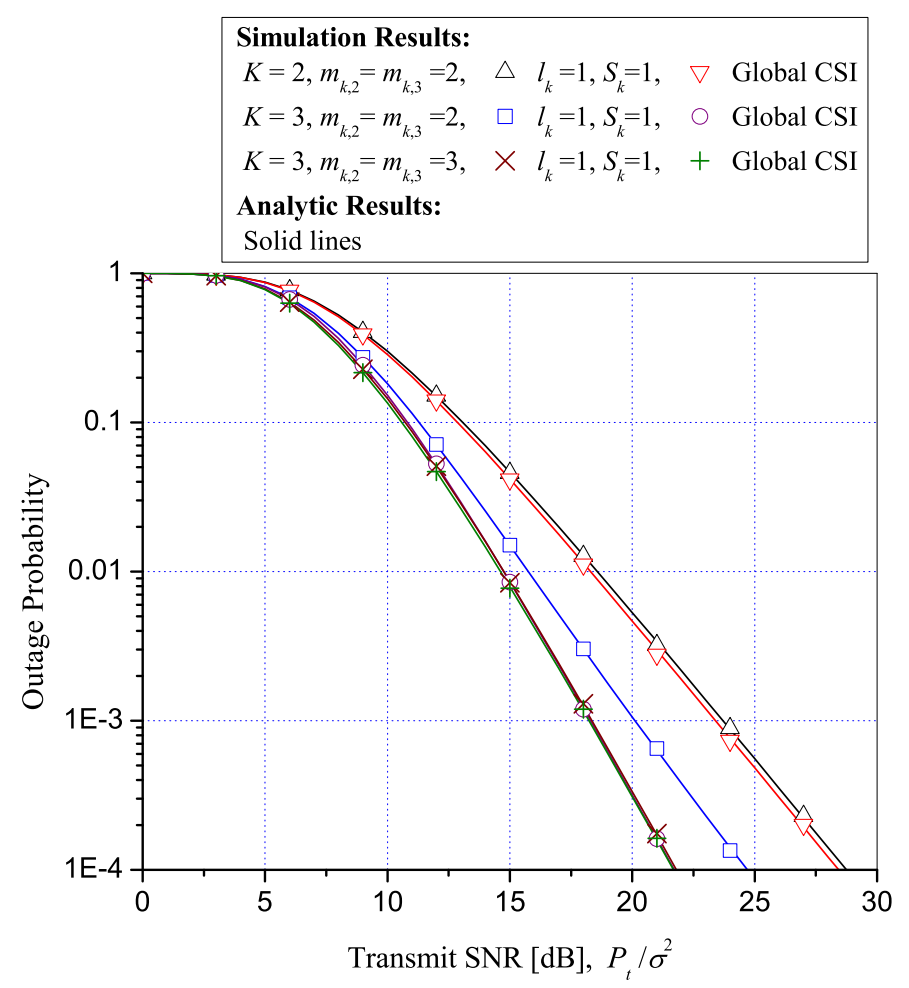

Figure 4. Outage probability for $m_{k, 1}=1, N_{k}=3$, and $l_{k}=1$ for all $k$ when $K=2,3$ and $R_{t}=1 \mathrm{bps} / \mathrm{Hz}$.

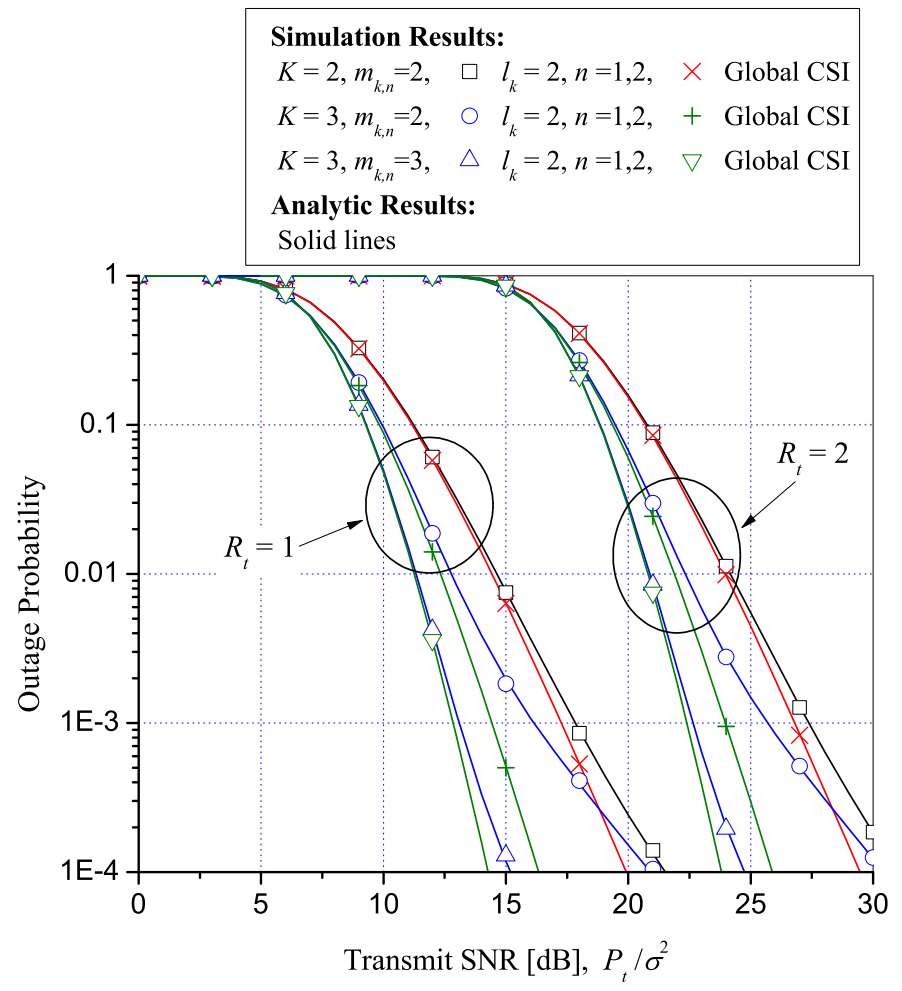

Figure 5. Outage probability for $m_{k, n}=2,3, N_{k}=3$, and $l_{k}=2$ for all $k$ and $n$ when $K=2,3$ and $R_{t}=1,2 \mathrm{bps} / \mathrm{Hz}$. 
Figures 6 and 7 compare the outage performances of the proposed DF-based PPS and the conventional AF-based PPS with $N_{k}=3$ for all $k, K=3$, and $R_{t}=1 \mathrm{bps} / \mathrm{Hz}$ when $P_{t} / \sigma^{2} \triangleq P_{k, n} / \sigma_{k, n}^{2}$ and $\beta_{k, n}=1$ for all $k$ and $n$, where it is noted that the average SNRs, $\beta_{k, n} P_{k, n} / \sigma_{k, n}^{2}$, are set to be equal for all $k$ and $n$, i.e., $\beta_{k, n} P_{k, n} / \sigma_{k, n}^{2}=P_{t} / \sigma^{2}$. By the traditional long-term routing process, the relays can be clustered relatively close together to establish the end-to-end communication from the source to the destination [33]. Thus, by the long-term routing process, there are $N-1$ clusters when $N_{k}=N$ for all $k$, where each cluster consists of $K$ relays. In addition, the long-term routing process results in the equivalent average SNRs for all the hops. Hence, in Figures 6 and 7, the setting of the same average SNR, $P_{t} / \sigma^{2}$, for all $k$ and $n$ is done by considering the long-term routing process. The routing method can monitor and utilize a change in large-scale fading such as path-loss and shadowing, but not that in small-scale fading. Thus, the proposed DF-based PPS and the conventional AF-based PPS [16] are allowed to use instantaneous CSI for path selection to track a change in small-scale fading. The conventional AF-based PPS uses only the first-hop CSI for path selection as well as AF relaying to minimize relay complexity. For the fair comparison, the proposed DF-based PPS with $l_{k}=1$ (i.e., one-hop CSI) is considered in the figures.

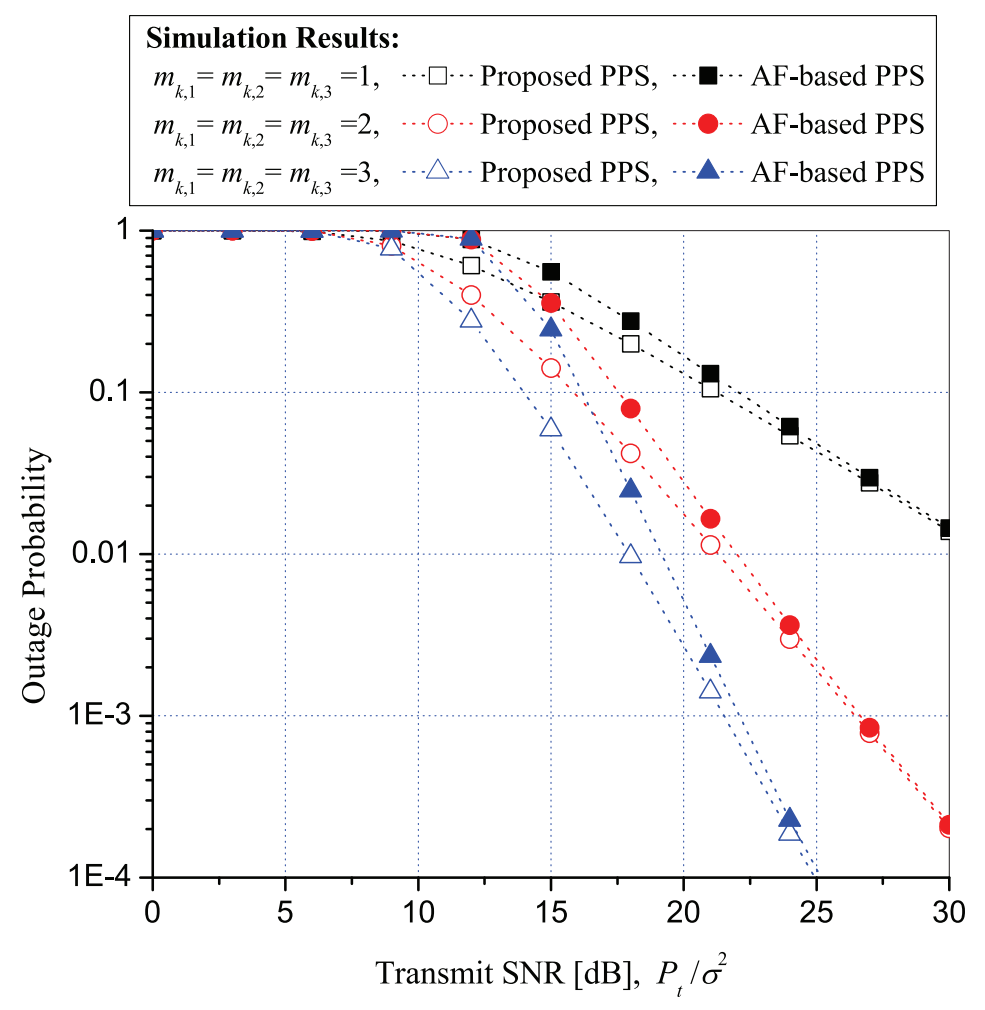

Figure 6. Outage probability of the proposed PPS with $l_{k}=1$ for all $k$ and the conventional AF-based PPS, when $m_{k, n}$ 's are equal, $N_{k}=3$ for all $k, K=3$, and $R_{t}=1 \mathrm{bps} / \mathrm{Hz}$. 


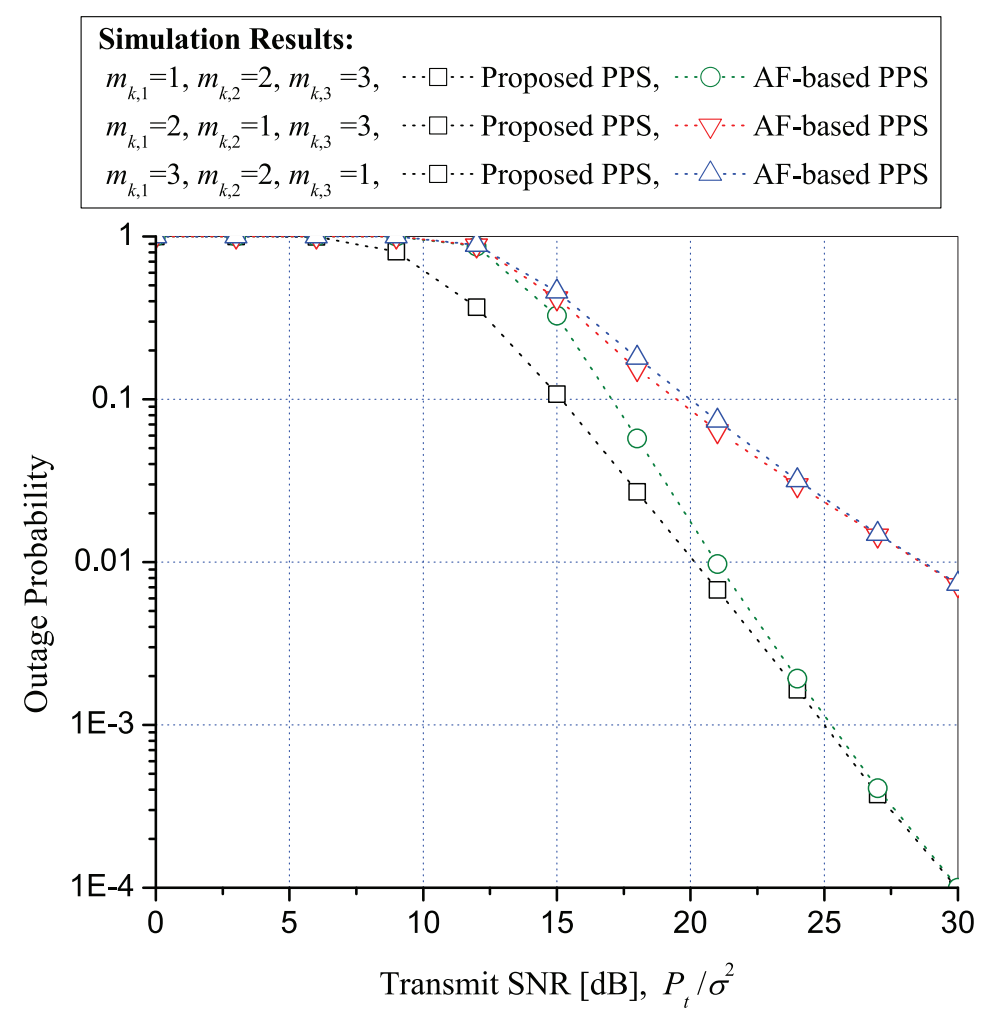

Figure 7. Outage probability of the proposed PPS with $l_{k}=1$ for all $k$ and the conventional AF-based PPS, when $m_{k, n}$ 's are unequal, $N_{k}=3$ for all $k, K=3$, and $R_{t}=1 \mathrm{bps} / \mathrm{Hz}$.

Figure 6 shows the outage performances of the proposed DF-based PPS and the conventional AF-based PPS when $m_{k, n}$ for all $k$ and $n$ are equal. In wireless channels, a line-of-sight component becomes stronger as the value of the Nakagami parameter $m_{k, n}$ increases. Hence, the outage performances of both PPS become better as $m_{k, n}$ rises. From the figure, it is observed that the proposed DF-based PPS works better than the conventional AF-based PPS in the low SNR regime, but both outage performances becomes similar for high SNR. The reason is that the noise propagation problem of AF relaying becomes severe for low SNR. As seen in Figure 6, the proposed PPS achieves no performance improvement in the high SNR regime, as compared with the conventional PPS, because the distributions of channels for all the hops are identical, and thus the one-hop CSI feedback for the both PPS works the same way. Therefore, in Figure 7, we assume unequal $m_{k, n}$ 's for all $n$ but equal for all $k$, which results in non-identical channel distributions for hops on each path. In the figure, there are the three non-identical cases: $m_{k, 1}=1, m_{k, 2}=2, m_{k, 3}=3 ; m_{k, 1}=2, m_{k, 2}=1$, $m_{k, 3}=3 ; m_{k, 1}=3, m_{k, 2}=2, m_{k, 3}=1$. For the three cases, the proposed PPS provides the same outage performance because the end-to-end channel distributions on each path are identical for all the three cases, and one-hop CSI feedback is done by selecting a hop with the maximum SNR correlation coefficient. However, the conventional PPS has different outage performances for the three cases since one-hop CSI feedback is always carried out using the first hop. It is noted that the conventional PPS does not change the first hop for CSI feedback, while the proposed PPS can change a hop for CSI feedback based on the SNR correlation coefficient. In the figure, when $m_{k, 1}=1, m_{k, 2}=2$, and $m_{k, 3}=3$, the performances of both PPS are similar for high SNR as the proposed PPS uses CSI feedback for the first hop like the conventional PPS. As shown in Figure 7, the proposed DF-based PPS generally attains considerable performance improvement as compared with the conventional AF-based PPS, although the proposed PPS requires more complexity for the CSI feedback process as well as DF relaying than the conventional PPS. 


\section{Conclusions}

For the MRS with the limited CSI feedback, the path selection using the end-to-end SNR correlation was newly introduced in this paper, and the exact and closed-form expression for the end-to-end SNR correlation coefficient was presented with only the Nakagami parameters and average SNRs for all the hops. Our proposed PPS with local CSI based on the highest end-to-end SNR correlation can achieve the most similar performance to the BPS with global CSI for a given limited feedback overhead. In particular, when the Nakagami parameters and the gap between average channel powers for hops are large, the PPS using CSI for only one of multiple hops can attain the analogous outage performance to the BPS using global CSI. The SNR correlation approach may be also applied to local CSI-based multi-hop cooperative systems such as multi-input multi-output relaying system, cognitive relaying system, NOMA-based relaying system, and physical-layer secure relaying system so as to improve their performances.

Author Contributions: The contributions of I.-H.L. were to propose the path selection scheme using the SNR correlation and analyze the SNR correlation coefficient and the end-to-end performance, while H.J. advised the ideas about the system model and the proposed scheme and suggested the mathematical analysis methodology and the simulation scenarios.

Funding: This work was supported by Basic Science Research Program through the National Research Foundation of Korea (NRF) funded by the Ministry of Education (Grant number: NRF-2018R1D1A1B07042499 and NRF-2016R1D1A1B03930060).

Conflicts of Interest: The authors declare no conflict of interest.

\section{Abbreviations}

The following abbreviations are used in this manuscript:

$\begin{array}{ll}\text { IoT } & \text { Internet of things } \\ \text { WSN } & \text { Wireless sensor network } \\ \text { BPS } & \text { Best path selection } \\ \text { PPS } & \text { Partial path selection } \\ \text { CSI } & \text { Channel state information } \\ \text { SNR } & \text { Signal-to-noise ratio } \\ \text { AF } & \text { Amplify-and-forward } \\ \text { DF } & \text { Decode-and-forward } \\ \text { NOMA } & \text { Non-orthogonal multiple access } \\ \text { MRS } & \text { Multi-hop parallel relay system } \\ \text { CDF } & \text { Cumulative distribution function } \\ \text { PDF } & \text { Probability density function }\end{array}$

\section{References}

1. Li, J.; Andrew, L.L.H.; Foh, C.H.; Zukerman, M.; Chen, H.H. Connectivity, coverage and placement in wireless sensor networks. Sensors 2009, 9, 7664-7693. [CrossRef] [PubMed]

2. Radi, M.; Dezfouli, B.; Bakar, K.A.; Lee, M. Multipath routing in wireless sensor networks: Survey and research challenges. Sensors 2012, 12, 650-685. [CrossRef] [PubMed]

3. Yick, J.; Mukherjee, B.; Ghosal, D. Wireless sensor network survey. Comput. Netw. 2008, 52, $2292-2330$. [CrossRef]

4. Akyildiz, I.F.; Su, W.; Sankarasubramaniam, Y.; Cayirci, E. Wireless sensor networks: A survey. Comput. Netw. 2002, 38, 393-422. [CrossRef]

5. Hasna, M.O.; Alouini, M.S. A performance study of dual-hop transmissions with fixed gain relays. IEEE Trans. Wirel. Commun. 2004, 3, 1963-1968. [CrossRef]

6. Hasna, M.O.; Alouini, M.S. Outage probability of multihop transmission over Nakagami fading channels. IEEE Commun. Lett. 2003, 7, 216-218. [CrossRef]

7. Nayak, P.; Vathasavai, B. Energy efficient clustering algorithm for multi-hop wireless sensor network using type-2 fuzzy logic. IEEE Sens. J. 2017, 17, 4492-4499. [CrossRef] 
8. Laneman, J.N.; Wornell, G.W. Distributed space-time-coded protocols for exploiting cooperative diversity in wireless networks. IEEE Trans. Inf. Theory 2003, 49, 2415-2425. [CrossRef]

9. Kim, J.B.; Kim, D. Exact and closed-form outage probability of opportunistic decode-and-forward relaying with unequal-power interferers. IEEE Trans. Wirel. Commun. 2010, 9, 3601-3606. [CrossRef]

10. Yu, H.; Lee, I.H.; Stüber, G.L. Outage probability of decode-and-forward cooperative relaying systems with co-channel interference. IEEE Trans. Wirel. Commun. 2012, 11, 266-274. [CrossRef]

11. Bletsas, A.; Khisti, A.; Reed, D.P.; Lippman, A. A simple cooperative diversity method based on network path selection. IEEE J. Select. Areas Commun. 2006, 24, 659-672. [CrossRef]

12. Su, X.; Sun, E.; Li, M.; Yu, F.R.; Zhang, Y. A survey on energy efficiency in cellular networks. Commun. Netw. 2013, 5, 654-660 [CrossRef]

13. Adigun, O.; Politis, C. Feedback information and energy efficiency of MIMO transmission modes in LTE. In Proceedings of the IEEE Sarnoff Symposium (SARNOFF), Newark, NJ, USA, 21-22 May 2012; pp. 1-5.

14. Xiong, C.; Li, G.Y.; Zhang, S.; Xu, S. CSI feedback reduction for energy-efficient downlink OFDMA. In Proceedings of the IEEE Wireless Communications and Networking Conference (WCNC), Shanghai, China, 1-4 April 2012; pp. 1135-1139.

15. Le, V.A.; Pitaval, R.A.; Blostein, S.D.; Riihonen, T.; Wichman, R. One-bit CSI feedback selection schemes for energy-efficient multiuser and multirelay systems. IEEE Trans. Wirel. Commun. 2013, 12, 1149-1161. [CrossRef]

16. Krikidis, I.; Thompson, J.; McLaughlin, S.; Goertz, N. Amplify-and-forward with partial relay selection. IEEE Commun. Lett. 2008, 12, 235-237. [CrossRef]

17. Lee, I.H. Outage performance of efficient partial relay selection in amplify-and-forward relaying systems over Rayleigh fading channels. IEEE Commun. Lett. 2012, 16, 1644-1647. [CrossRef]

18. Kundu, C.; Ghose, S.; Bose, R. Secrecy outage of dual-hop regenerative multi-relay system with relay selection. IEEE Trans. Wirel. Commun. 2015, 14, 4614-4625. [CrossRef]

19. Jaafar, W.; Ohtsuki, T.; Ajib, W.; Haccoun, D. Impact of the CSI on the performance of cognitive relay networks with partial relay selection. IEEE Trans. Veh. Technol. 2016, 65, 673-684. [CrossRef]

20. Lee, S.; da Costa, D.B.; Vien, Q.T.; Duong, T.Q.; de Sousa, R.T. Non-orthogonal multiple access schemes with partial relay selection. IET Commun. 2017, 11, 846-854. [CrossRef]

21. Ding, Z.; Dai, H.; Poor, H.V. Relay selection for cooperative NOMA. IEEE Wirel. Commun. Lett. 2016, 5, 416-419. [CrossRef]

22. Vien, Q.T.; Nguyen, H.X.; Shah, P.; Ever, E.; To, D. Relay selection for efficient HARQ-IR protocols in relay-assisted multisource multicast networks. In Proceedings of the IEEE Vehicular Technology Conference (VTC), Seoul, Korea, 18-21 May 2014; pp. 1-5.

23. Bhatnagar, M.R.; Mallik, R.K.; Tirkkonen, O. Performance evaluation of best-path selection in a multihop decode-and-forward cooperative system. IEEE Trans. Veh. Technol. 2016, 65, 2722-2728. [CrossRef]

24. Bhatnagar, M.R. Performance analysis of max-min path selection scheme in multi-hop DF cooperative system over Nakagami- $m$ channels. In Proceedings of the 2014 International Conference on Signal Processing and Communications (SPCOM), Bangalore, India, 22-25 July 2014; pp. 1-5.

25. Lee, I.H.; Lee, S. Bit error probability of decode-and-forward relaying with efficient partial relay selection in non-identical Rayleigh fading channels. IET Commun. 2014, 8, 2624-2632. [CrossRef]

26. Lee, S.; Lee, H.; Choi, H.H.; Lee, I.H. Outage probability of decode-and-forward relaying systems with efficient partial relay selection in Nakagami fading channels. ETRI J. 2014, 36, 22-30. [CrossRef]

27. Michalopoulos, D.S.; Suraweera, H.A.; Karagiannidis, G.K.; Schober, R. Amplify-and-forward relay selection with outdated channel estimates. IEEE Trans. Commun. 2012, 60, 1278-1290. [CrossRef]

28. Soysa, M.; Suraweera, H.A.; Tellambura, C.; Garg, H.K. Partial and opportunistic relay selection with outdated channel estimates. IEEE Trans. Commun. 2012, 60, 840-850. [CrossRef]

29. Kim, J.B.; Lee, I.H. Outage analysis of partial relay selection schemes with feedback delay and channel estimation errors in nonidentical Rayleigh fading channels. Int. J. Ant. Propag. 2017, 2017, 1-9. [CrossRef]

30. Gradshteyn, I.S.; Ryzhik, I.M. Table of Integrals, Series, and Products, 7th ed.; Academic Press: Burlington, VT, USA, 2007.

31. Lee, I.H.; Kim, D. Outage probability of multi-hop MIMO relaying with transmit antenna selection and ideal relay gain over Rayleigh fading channels. IEEE Trans. Commun. 2009, 57, 357-360. [CrossRef] 
32. Lee, I.H.; Kim, D. Spatial channel reuse in multi-hop decode-and-forward relaying systems in high path loss environments. IEEE Commun. Lett. 2012, 16, 990-993. [CrossRef]

33. Karaki, J.N.A.; Kamal, A.E. Routing techniques in wireless sensor networks: a survey. IEEE Wirel. Commun. 2004, 11, 6-28. [CrossRef] 